\title{
Neue, erfolgreiche Therapie beim venösen Ulcus cruris mit der segmental- adaptiven Kompressionsmanschette (SAKM) ${ }^{*}$
}

\author{
New Successful Therapy for Venous Ulcus Cruris Using the Segmental Adaptive Compression Sleeve
}

Autoren

Institut
A. Hechmat-Dehkordi, T. Möhler, B. Kleis-Fischer, S. Grabbe, R. Schopf

Hautklinik, Universitätsmedizin Mainz

\section{Bibliografie}

DOI http://dx.doi.org/

$10.1055 / \mathrm{s}-0030-1255864$

Online-Publikation: 26. 10. 2010

Akt Dermatol 2010; 36:

474-479 @ Georg Thieme

Verlag KG Stuttgart · New York ISSN 0340-2541

\section{Korrespondenzadresse}

Dr. med. Abolghassem Hechmat-Dehkordi

Hautklinik,

Universitätsmedizin Mainz

Hegelstraße 70

55122 Mainz

dr.hechmat@gmx.de

\section{Zusammenfassung \\ V}

Hintergrund: Publikationen zeigen, dass die herkömmliche Kompressionstherapie bei chronisch venöser Insuffizienz nur in weniger als $47 \%$ der Fälle bei Kompressionsstrumpfanwendung und $31 \%$ der Fälle bei Kompressionsverbandanwendung zu der gewünschten Ödemreduktion und Abheilung des Ulcus cruris führt. Daher sind andere Therapieformen wünschenswert.

Patienten und Methodik: Wir stellen ein Verfahren für therapierefraktäre Ulcera crurum mittels täglich segmental anpassbarem Kompressionsdruck vor. Wir behandelten 25 Patienten mit chronisch-venöser Insuffizienz sowie Unterschenkelödemen, von denen 13 ein nicht heilendes Ulcus cruris hatten, nach einer Vorbehandlung von Jahrzehnten (1-40 Jahre) über einen Zeitraum von etwa 2 - 8 Monaten. Die Patienten trugen eine segmental-zirkulär-adaptiv dorsal geschlossene Unterschenkel-Kompressionsmanschette. Im Fußrücken und Knöchelbereich hat die Manschette sechs $2 \mathrm{~cm}$ breite Bänder mit Klettverschluss, pro-

\section{Einleitung}

1,5 bis 2 Millionen Menschen leiden in Deutschland an einem Ulcus cruris: In der 2003 publizierten Bonner Venenstudie lag die Prävalenz für das Vorliegen eines abgeheilten Ulcus cruris bei 0,6\% (2,4\% in der Altersgruppe 70-79 Jahre) und bei $0,1 \%$ ( $0,3 \%$ bei den 70 - bis 79 -Jährigen) für das Vorliegen eines aktiven Ulcus cruris [1].

Etwa $70 \%$ der Ulzera sind venös bedingt, $10 \%$ arterieller Genese, 10\% gemischt arterio-venös und lediglich zirka $10 \%$ sind anderer Genese [2]. Insuffiziente Klappenventile können die unidirektional, herzwärts gerichtete Strömung des Blutes nicht gewährleisten. So fließt das Blut peripherwärts. Die Kapillaren werden erweitert und vermehrt geschädigt. Aus den geschädigten Kapilla- ximal davon bis unterhalb des Knies fünf Schlaufen mit einer Breite von $5 \mathrm{~cm}$, die durch Zugkraft einen bedarfsweise angepassten Kompressionsdruck erzeugen. Dieser Kompressionsdruck ist für jedes Segment getrennt täglich variabel einstellbar. Der Beinumfang wurde in den folgenden 1 - 17 Tagen verglichen. Die Akzeptanz wurde mittels visueller Analogskala geprüft.

Ergebnisse: Nach einem Tag bestimmten wir im Median folgende verminderte Beinumfänge in 4 Bereichen: 53 auf $48 \mathrm{~cm}$ (Fußrücken), 44 auf $43 \mathrm{~cm}$ (Knöchel), 36 auf $33 \mathrm{~cm}$ (Wade), 27 auf $26 \mathrm{~cm}$ (unterhalb der Knie). In den folgenden 117 Tagen blieb der Beinumfang in allen fünf Bereichen konstant vermindert, ohne Ödembildung. Die Akzeptanz des Verfahrens war bei den Patienten sehr groß.

Schlussfolgerung: Unsere Befunde zeigen, dass die wirksame Verdrängung des Ödems mittels täglich segmental anpassbarem Kompressionsdruck innerhalb von 2-8 Monaten zur Abheilung des jahrzehntelang bestehenden Ulcus crurum führt.

Liegt ein Phlebödem vor, so ist die medizinische Kompressionstherapie zur Behebung des Ödems unabdingbar. Ohne Ödemreduktion ist eine Wundheilung nicht möglich. Das Ödem behindert die Kapillarzirkulation. Die Diffusionsstrecke für die Nährstoffe im Gewebe wird so stark erhöht, dass eine ausreichende Nährstoffversorgung der Körperzellen nicht mehr gewährleistet ist.

\footnotetext{
* Daten des Abstracts z.T. vorgetragen auf der DDG, Dresden 2009 .
}

ren sammelt sich im interstitiellen Raum Ödemflüssigkeit an. An Stellen mit besonders ungünstigen hämodynamischen Veränderungen entwickelt sich eine schwere Mikroangiopathie: Ulcus 

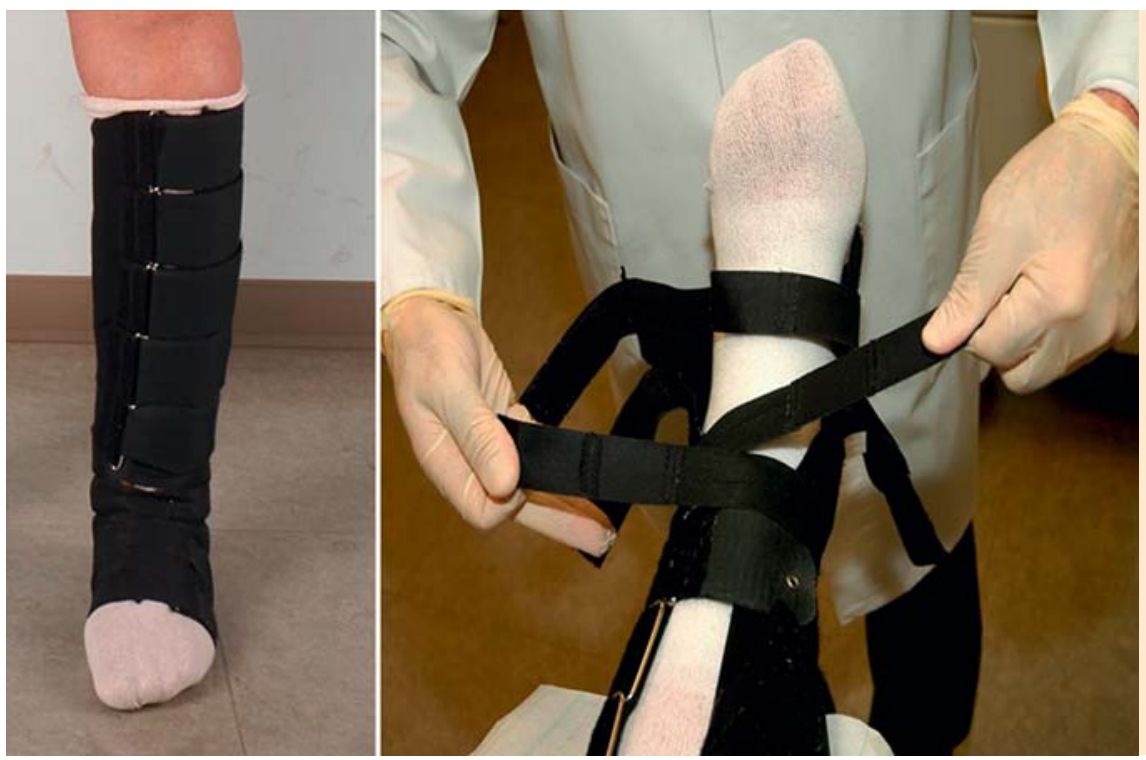

Abb. 1 Segmental-adaptive Kompressionsmanschette (SACM).

Die medizinische Kompressionstherapie kann dabei entweder mit Kompressionsstrümpfen oder Kompressionsverbänden mit Kurzzugbinden und intermittierenden Methoden durchgeführt werden [3]. Bei dieser Therapie sind insbesondere ältere Patienten auf Hilfe angewiesen. Das Verhältnis vom Fersenumfang zum Supramalleoloarbereich ist $3: 2$. Dies bedeutet, dass der Patient den engsten Teil des Strumpfes über einen bedeutend weiteren Umfang ziehen muss. Sitzt der Strumpf nicht richtig, so kann es durch Faltenbildung und Strangulierung zu einer Verschlechterung der venösen Situation am Bein führen. Hier kann eine Hauptursache für das häufige Rezidiv nach abgeheiltem Ulcus cruris liegen. Auch bei den Kompressionsverbänden können Lockerungen und Lückenbildung zu einer unzureichenden Kompression führen [4].

Da die herkömmlichen Kompressionsmethoden oftmals nicht zu einer ausreichenden Ödemreduktion bis zur Heilung des Ulcus cruris führen, haben wir das neue Therapieverfahren mit segmental-adaptiver Kompressionsmanschette (SAKM) entwickelt (๑ Abb. 1).

\section{Patienten und Methode \\ $\nabla$}

Die therapeutische Studie wurde von der zuständigen Ethikkommission genehmigt.

Die Patienten wurden ausführlich aufgeklärt und gaben ihre Zustimmung zu der dargestellten Therapie. Eingeschlossen wurden Patienten mit chronisch venöser Insuffizienz (diagnostiziert durch klinische Untersuchung und Duplexsonografie mit einem Schweregrad entsprechend der CEAP-Einteilung (clinic, etiology, anatomy, pathophysiology) von mindestens C3 (Ödem) sowie Patienten mit sekundärem Ödem. Ausgeschlossen wurden Patienten mit einer relevanten peripheren arteriellen Verschlusskrankheit (pAVK) (Knöchel-Arm-Index <0,8) und einem Diabetes mellitus (HbA1c > 6,5\%).

Durch die segmental-adaptive Kompressionsmanschette, die im Fußrücken und Knöchelbereich sechs $2 \mathrm{~cm}$ breite Bänder mit Klettverschluss und proximal davon bis unterhalb des Knies fünf $5 \mathrm{~cm}$ breite Schlaufen hat, ist es möglich, einen durch Zugkraft bedarfsweise angepassten Kompressionsdruck unter jedem zirkulären Segment zu erzeugen.
Tab. 1 Unterschiede zwischen dem Kompressionsstrumpf und der Kompressionsmanschette.

\begin{tabular}{lcc} 
Besonderheiten & $\begin{array}{c}\text { Kompressions- } \\
\text { strumpf }\end{array}$ & $\begin{array}{c}\text { segmental-adaptive } \\
\text { Kompressionsmanschette }\end{array}$ \\
$\begin{array}{l}\text { Strangulierung } \\
\begin{array}{l}\text { Ulcus cruris } \\
\text { venosum }\end{array}\end{array}$ & ++ & - \\
\hline $\begin{array}{l}\text { Anziehen/Ausziehen } \\
\text { Effektivität beim }\end{array}$ & - & ++ \\
Lymphödem & $+/-$ & ++ \\
$\begin{array}{l}\text { tägliche adaptive } \\
\text { Kompression }\end{array}$ & - & ++ \\
\hline
\end{tabular}

Die Wundauflage wird vor der Anlage der segmental-adaptiven Kompressionsmanschette individuell angepasst. Danach wird am Unterschenkel ein Tg-Schlauch angebracht.

Die Manschette wird am Unterschenkel mittels Bändern locker angelegt. Danach erfolgt die Einstellung des Kompressionsdruckes jedes Bandes, angefangen von der Fußspitze bis zum Knie.

Der Kompressionsdruck ist an der Fußspitze höher als im Kniebereich. Der Übergang des höheren Kompressionsdrucks zum niedrigen erfolgt segmental, aber kontinuierlich.

Am sitzenden Patienten wird die Manschette angelegt und der Kompressionsdruck wird manuell für jedes Segment eingestellt. Der Druck unter jedem Segment wird in Abstimmung der Druckwahrnehmung des Arztes und der Toleranz des Patienten festgesetzt. Der jeweilige Druck wird auf dem zugehörigen Klettband für den Patienten verständlich markiert. Diese Einstellungen behält der Patient für die weiteren Tage bis zum nächsten Arztbesuch bei. Nach anfänglicher Schulungs- und Kontrollphase durch den behandelnden Arzt kann der Patient dann in der Regel selbstständig mit der Manschette umgehen.

Nach Aufklärung der Patienten über die Funktionsweise der Manschette wurden die Beinumfänge an folgenden Stellen gemessen: unterhalb Knie, im Gastrocnemiusbereich, supramalleolär und metatarsal.

Im weiteren Verlauf wurden die Patienten über den Tragekomfort, aufgetretene Schmerzen, Spannungsgefühl, Druckstellen/ Wärmestau/Ekzembildung und die Handhabung der Manschette 


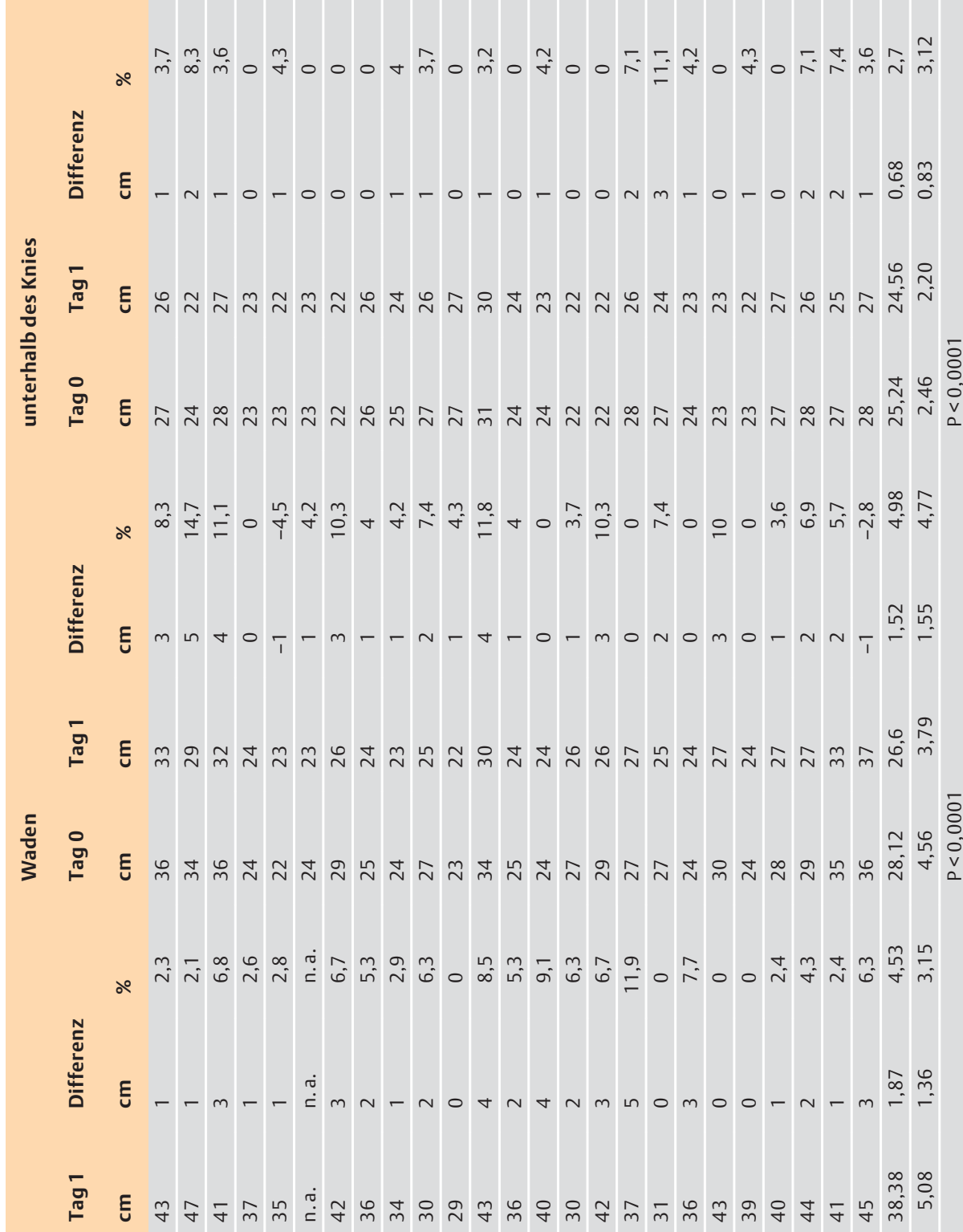

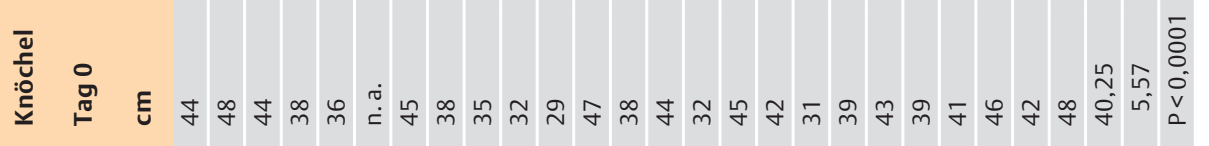

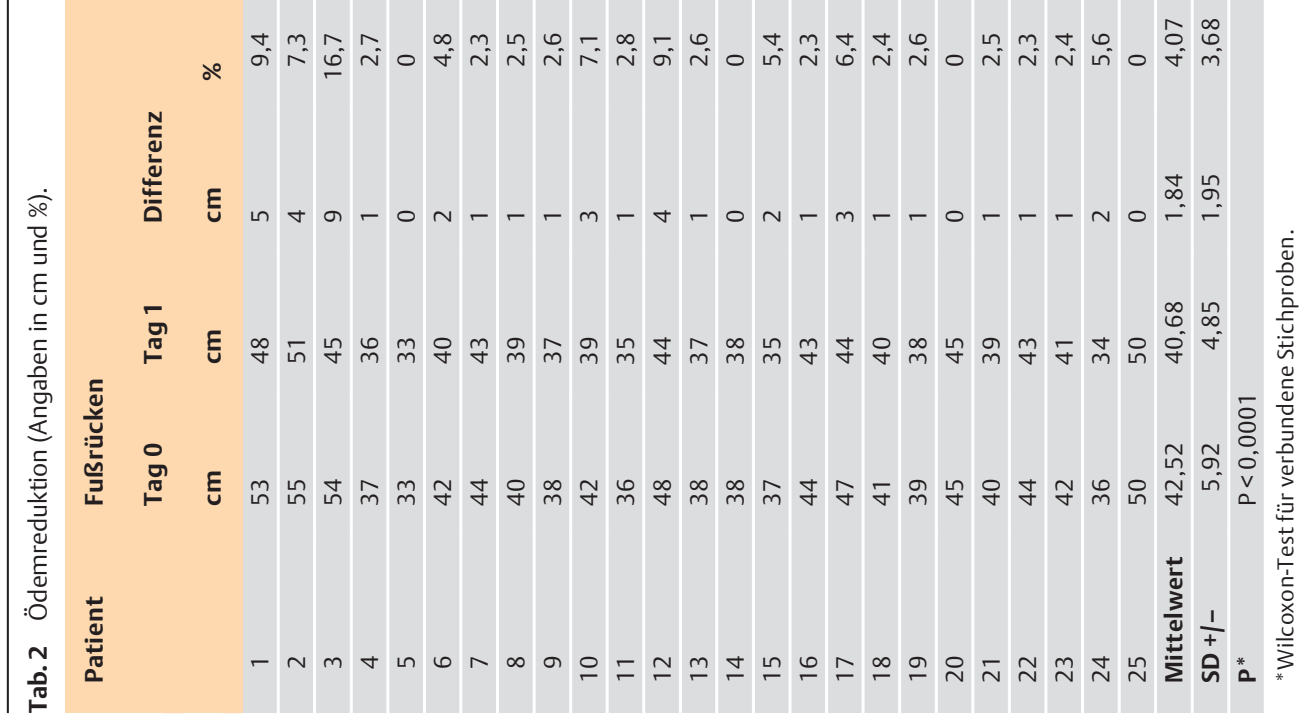



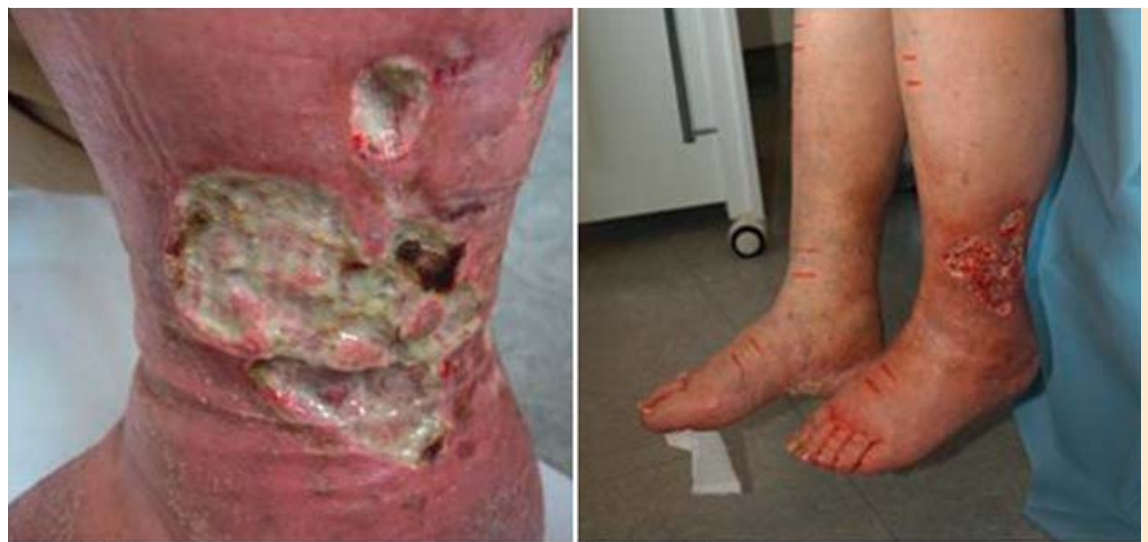

Abb. 2 Abheilung eines Ulcus cruris unter Therapie mit segmental-adaptiver Kompressionsman-
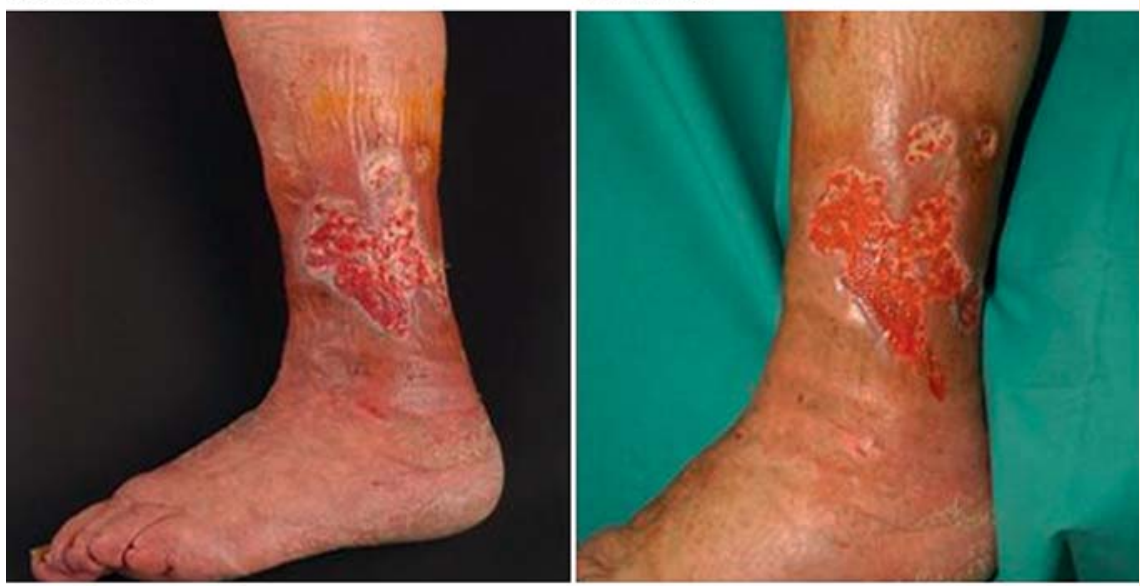

schette.

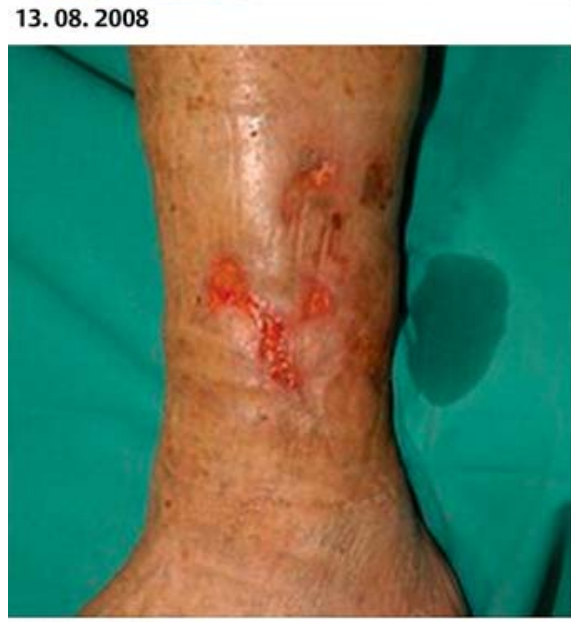

20.08. 2008

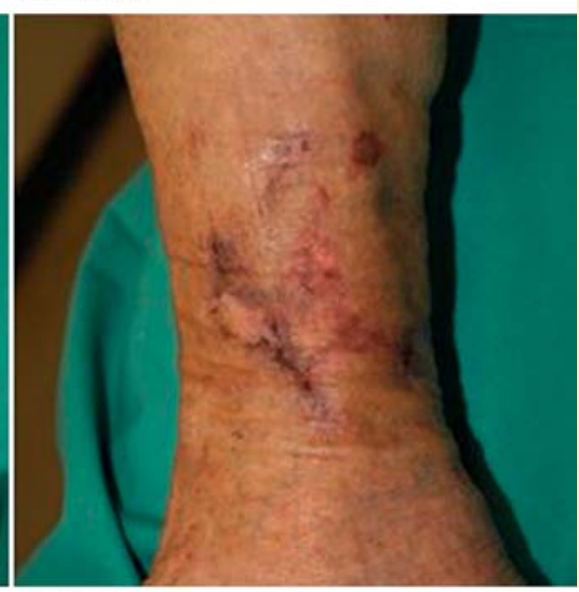

09. 10.2008

02. 12.2008

mittels visueller Analogskala nach bestimmten Kriterien befragt (๑ Tab.4).

Die Umfänge wurden an den genannten Stellen gemessen, immer in sitzender Position und nachmittags um 14 Uhr vom selben Untersucher, um Schwankungen der Messwerte gering zu halten (๑ Tab. 2).

Bei wöchentlicher Einbestellung der Patienten mit Ulcus cruris wurde der Beinumfang bis zur Abheilung der Ulzeration ermittelt. Die Messwerte wurden statistisch mit dem Wilcoxon-Test für verbundene Stichproben berechnet ( $\square$ Tab. 2). Außerdem erfolgte immer eine Fotodokumentation.

Patienten mit doppelseitigen Ulcus cruris waren in unserem Patientengut nicht vertreten.

\section{Ergebnisse}

\section{$\nabla$}

1. Rasche Ödem-Reduktion:

Der Beinumfang verminderte sich innerhalb der ersten 24 Stunden und blieb dann über die folgenden 7-17 Tage konstant ( Tab. 2).

2. Verkürzung der Therapiedauer:

Die jahrzehntelang ( 1 - 40 Jahre) anhaltenden Ulzera heilten innerhalb von 2-8 Monaten ab ( Tab. 3).

3. Akzeptanz der Patienten:

Die Patienten berichten, dass die Therapieform äußerst angenehm und entspannend ist. Anhand der visuellen Analogskala berichten die Patienten, dass sie die segmental-adaptive Kompressionsmanschette bevorzugen ( $\bullet$ Tab. 4). 
Tab.3 Therapiedauer mit segmental-adaptiver Kompressionsmanschette bei Ulcus cruris.

\begin{tabular}{|c|l|l|}
\hline Patient & $\begin{array}{c}\text { therapierefraktäre } \\
\text { Jahre ohne Erfolg }\end{array}$ & Therapiedauer \\
\hline 1 & 1 & $\mathbf{6}$ Monate \\
\hline 2 & 36 & $\mathbf{7}$ Monate \\
\hline 3 & 38 & $\mathbf{7}$ Monate \\
\hline 4 & 1 & $\mathbf{4}$ Monate \\
\hline 5 & 1 & $\mathbf{3 , 5}$ Monate \\
\hline 6 & 15 & $\mathbf{2}$ Monate \\
\hline 7 & 2,5 & $\mathbf{3}$ Monate \\
\hline 8 & 1 & $\mathbf{1 , 5}$ Monate \\
\hline 9 & 1 & $\mathbf{2 , 2}$ Monate \\
\hline 10 & 40 & $\mathbf{7 , 5}$ Monate \\
\hline 11 & 27 & $\mathbf{2}$ Monate \\
\hline 12 & 1 & $\mathbf{3}$ Monate \\
\hline 13 & 40 & $\mathbf{8}$ Monate \\
\hline
\end{tabular}

Tab. 4 Patientenakzeptanz bei zehn Patienten: Bedienbarkeit und Tragekomfort.

\begin{tabular}{|c|c|c|c|}
\hline Patient & $\begin{array}{l}\text { Kompressions- } \\
\text { strumpf }\end{array}$ & $\begin{array}{l}\text { Kurzzug- } \\
\text { binde }\end{array}$ & $\begin{array}{l}\text { segmental-adaptive } \\
\text { Kompressions- } \\
\text { manschette }\end{array}$ \\
\hline 1 & 5 & 6 & 10 \\
\hline 2 & 7 & 7 & 9 \\
\hline 3 & 5 & 8 & 10 \\
\hline 4 & 4 & 7 & 9 \\
\hline 5 & 5 & 6 & 9 \\
\hline 6 & 4 & 6 & 10 \\
\hline 7 & 5 & 7 & 9 \\
\hline 8 & 6 & 7 & 10 \\
\hline 9 & 6 & 5 & 10 \\
\hline 10 & 5 & 8 & 10 \\
\hline Summe & 52 & 67 & 96 \\
\hline
\end{tabular}

Patientenaussage in visueller Analogskala von $0-10$

Im Vergleich zu Kompressionsstrumpf und Kurzzugbinde bevorzugen alle befragten Patienten die segmental-adaptive Kompressionsmanschette ( $\bullet$ Tab. 1 und 4).

Die Fotos zeigen die positiven Wirkungen der segmental-adaptiven Kompressionsmanschette $(\bullet$ Abb. 2).

\section{Diskussion}

Viele Patienten mit einer CVI sind nicht in der Lage, die medizinisch notwendigen Kompressionsstrümpfe alleine anzuziehen. Gerade die zum Teil atrophe und leicht verletzbare Haut am Innenknöchel kann beim gewaltsamen Anziehen der Kompressionsstrümpfe zusätzlichen Schaden erleiden. Neben der geschädigten Haut haben wir in diesem Bereich auch die im Vergleich zur Wade fehlende Muskelpumpe und einen nachlassenden Kompressionsdruck der Strümpfe. Die distale Kompressionswirkung lässt bei unverändertem Kompressionsdruck im proximalen Anteil so stark nach, dass ein gegenteiliger Effekt entstehen und es zu einer Stauung im Knöchelbereich kommen kann, einer möglichen Ursache von rezidivierendem Ulcus cruris [7].

Zur Lösung dieses Problems wurde von uns die segmental-adaptive Kompressionsmanschette entwickelt, deren Handhabung für den Patienten einfach ist. Die Kompressionsmanschette ist mit geringem Kraftaufwand sehr benutzerfreundlich. Es wird eine effektive und rasche Ödemreduktion erreicht.

Da die Beinlänge der Patienten unterschiedlich ist, wird die Anzahl der Segmentalbänder nach der jeweiligen Beinlänge des Patienten bestimmt.

Die Patienten wurden zu den herkömmlichen Therapieverfahren mittels Kompressionsbestrumpfung und/oder Kurzzugbinden im Vergleich zur SAKM befragt ( Tab. 4).

Neben einem jahrzehntelangen Krankheitsverlauf der betroffenen Patienten waren im Vorfeld konventionelle Therapiemaßnahmen ausgeschöpft worden (Varizensanierung, moderne Wundtherapie, Ulkusshaving, Meshgraft, Vakuum-Therapie [5]). Wir zeigen mit der vorliegenden Arbeit den therapeutischen Vorteil der segmental-adaptiven Kompressionsmanschette gegenüber den herkömmlichen Kompressionsmethoden, insbesondere die Verkürzung der Behandlungsdauer auf $1-8$ Monate $($ Tab. 1 und 3).

In weiteren Studien (Messung der Anpressdrücke, volumetrische 3-D-Messung der Beine) muss die Wirksamkeit der segmentaladaptiven Kompressionsmanschette (SAKM) näher erforscht werden. Ebenso müssen vergleichende Studien folgen, in denen Ulkuspatienten nach den Regeln der modernen Wundversorgung, jedoch mit unterschiedlichen Kompressionssystemen (Kurzzugbinde, Strumpf, SAKM) behandelt werden, um den Erfolg der SAKM weiter zu eruieren.

\section{Abstract}

\section{New Successful Therapy for Venous Ulcus Cruris Using the Segmental Adaptive Compression Sleeve \\ $\nabla$}

Background: Publications demonstrate that conventional compression therapy in less than $47 \%$ of cases with surgical stocking and less than $31 \%$ with surgical bandage leads to the required edema reduction in chronic venous insufficiency. Therefore other forms of treatment are required.

Patients and methods: We present a procedure for treatment-resistant leg ulcers using daily adaptable compression. Twenty-five patients who had chronic venous insufficiency with non-healing leg ulcers and/or massive edema over a period of several decades were treated for a period of about 5 months. The patients wore a segmental-circular-adaptive lower leg sleeve closed dorsally. At the dorsum of the foot and in the ankle area the sleeve has six $2 \mathrm{~cm}$ wide tapes with Velcro fasteners, in the proximal area until below the knee it has five $5 \mathrm{~cm}$ wide straps which generate a compression by tensile force that is adaptable as required. This compression can be adjusted daily for each segment.

Results: After 1 day, the median of the following reduced leg circumferences were achieved in 4 areas: from 53 to $48 \mathrm{~cm}$ (dorsum of the foot), from 44 to $43 \mathrm{~cm}$ (ankle), from 36 to $33 \mathrm{~cm}$ (calve), from 27 to 26 (below the knee). In case of all patients with therapy resistant necrotizing, progressive ulcerations, these were completely healed. The acceptance of the procedure with the patients was very good.

Conclusion: The precise segmental compression sleeve leads to the heeling of therapyresistent venous leg ulcers. 


\section{Literatur}

1 Pannier-Fischer F, Rabe E. Epidemiologie der chronischen Venenerkrankungen. Hautarzt 2003 Nov; 54 (11): 1037-1044

2 Partsch $H$. Klassifizierung und Bewertung von chronischen Venenerkrankungen der unteren Extremitäten. Phlebol 1995; 24: 125-129

3 Hoffmann U, Tató F. Periphere Zirkulation. In: Siegenthaler W, Blum HE. Klinische Pathophysiologie, 9. Auflage. Stuttgart: Thieme, 2006: $695-750$
4 Milic DJ, Zivic SS, Bogdanovic DC et al. Risk factors relted to the failure of venous leg ulcers to heal with compression treatment. J Vasc Surg 2009; 49: 1242 - 1247

5 Coleridge-Smith PD. Leg ulcer treatment. J Vasc Surg 2009; 49: 804-808

6 Gaber Y. Erkrankungen der Lymphgefäße. In: Braun-Falco O et al. Dermatologie und Venerologie, 5. Auflage. Heidelberg: Springer, 2005: $829-834$

7 Finlayson $K$, Edwards $H$, Courtney $M$. Factors associated with recurrence of venous leg ulcers: a survey and retrospective chart review. Int J Nurs Stud 2009 Aug; 46 (8): 1071 - 1078. Epub 2009 Jan 30 